\title{
Forensic Engineering Analysis of Impacts to the Adult and Pediatric Head
}

by Laura L. Liptai, Ph.D. (NAFE 339C)

\begin{abstract}
This reports forensic engineering analysis of impacts to the adult head and pediatric head from multiple sources, with the goal of amassing a database to be utilized for Forensic BioMedical Engineering analysis. Supplemental impact activities (of acceleration verses time data), rather than cumulative historical adult and child head impacts are discussed. Adult head impacts recently tested include motorcycle, bicycle and equestrian helmeted impacts. Infant head impacts include falls onto carpet/tile and toy impacts, and child head impacts include recreational ball tests (baseballs, soccer balls, playground balls and basketballs).
\end{abstract}

BioMedical engineers use computational modeling and simulation to analyze injurious trauma to the human head and brain. The verification of these mathematical models, codes, solutions, and/or simulations depends upon the quality of their experimental validation. The reliability of the experimental data determines the validity of the modeling. A database of real world head and brain impact responses is constructed utilizing principals from biomedical engineering, biomechanics and anthropometric dummy testing. The digital data is collected according to a standardized protocol using a tri-axial accelerometer mounted inside of various anthropometric dummy craniums of infants, children and adults. The performance of motorcycle, bicycle and equestrian helmet types is analyzed with some unexpected results. Other data includes pediatric experimental results that have not been previously reported: child head impacts with various sports equipment, infant head impacts to surfaces commonly in the home, like tile and carpet, as well as infant head impacts with massive, hard plastic toys. The objective is to quantify the pediatric and adult human head responses in the form of acceleration verses time data to impact and non-impact scenarios. This data will supplement the forensic engineering experimental analysis database as well as define variables utilized in the mathematical modeling of injurious head impacts to improve head impact safety counter measures.

\section{Keywords}

Head Impact, Helmets, Motorcycle, Bicycle, Sports Equipment, Head Impact, Infant Head, Child Head, Head Trauma, Brain Injury, Testing, BioMedical Engineering, Biomechanics, Equestrian Helmets, Ball to Head Impacts, Head Impact onto Tile, Head Impact onto Carpet 


\section{Introduction}

Head impacts are a relatively frequent occurrence in the human experience where a proportion of persons sustain serious brain injury. Motorcyclists encounter many different impact configurations in collisions. Infants are accidentally dropped onto surfaces with non-yielding characteristics; children fall while playing sports and other daily events. For some activities it is commonplace to take precautions against head injuries with countermeasures like helmets. Other impacts, even with repeat exposures, like heading the ball in soccer, may not utilize safety equipment. We are comparing a wide variety of countermeasures as well as impacts without protection. A variety of head impacts under various forensic circumstances are analyzed and the likelihood of diffuse brain trauma is assessed utilizing federally standardized protocols. This data can then be utilized to improve mathematical models of head impact and to analyze countermeasures in real world performance, beyond standardized testing.

\section{Countermeasure Design}

The analysis reveals that countermeasures are generally designed to be optimized for one exposure profile. If the impact is within this exposure profile, the protective response is improved. If the real world impact is outside of the designed exposure profile, this is clearly evident in the results. For example, the motorcycle helmet may not perform as well as a bicycle helmet in lower velocity impacts. However, a bicycle helmet in a higher velocity collision could be catastrophic. It is thought that the biological material properties of the skull are similarly optimized; some theorize that the linear skull fracture absorbs the energy that would have been experienced by the brain.

\section{Theoretical Analysis of Impact}

No single physical variable can determine the severity of impact to the human brain. There are a few core factors that determine the severity of an impact; the associated speeds just before and after impact, the elasticity and mass of the two colliding bodies, the stress strain ratio of the material(s), and the duration of the impact. In the event of permanent deformation like a fracture, the inelasticity of the bodies may also be considered in the coefficient of restitution $(\varepsilon)$. The coefficient of restitution is a common measure of impact plasticity in models using the conservation of momentum principals to deduce final velocities (Figure 1). With permanent deformation comes a loss in kinetic energy of the system. In a two bodied inelastic collision, this loss

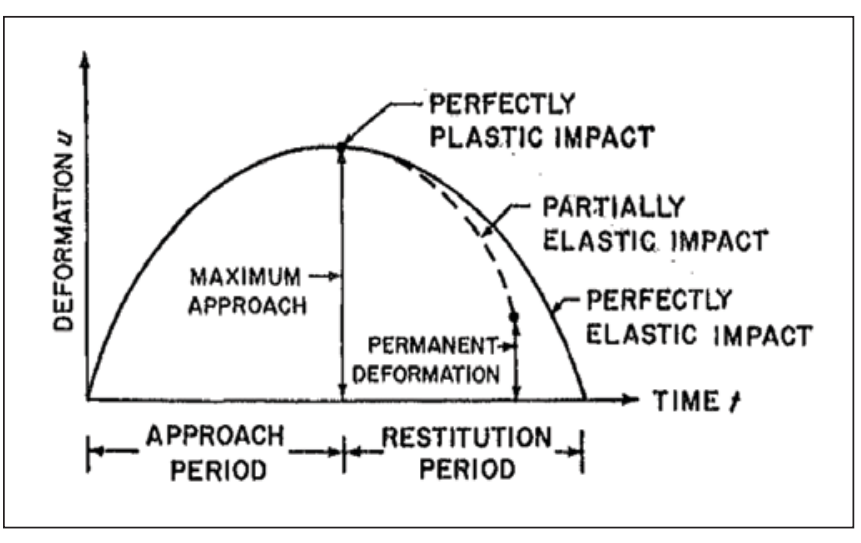

Figure 1

Deformation in Stereomechanical Impact (9)

of kinetic energy of bodies with velocities normal to impact $\left(\mathrm{v}_{\mathrm{n}}\right)$ is given by Equation 1 . 
$\Delta T=\frac{1}{2}\left(\frac{m_{1} m_{2}}{m_{1}+m_{2}}\right)\left(1-\varepsilon^{2}\right)\left(v_{1, n 0}-v_{2, n 0}\right)^{2}$

Equation 1

$\Delta I \bar{\omega}=I \dot{\omega}-I \dot{\omega} 0=\int_{0}^{t} \overline{F r} d t$

Equation 3

$$
\Delta m \vec{v}=m \vec{v}-m \overrightarrow{v_{0}}=\int_{0}^{t} \vec{F} d t
$$

Equation 2

$$
\mathrm{HIC}=\left\{\frac{1}{\left(t_{2}-t_{1}\right)} \int_{t_{1}}^{t_{2}} a d t\right\}^{2.5}\left(t_{2}-t_{1}\right)<1000
$$

\section{Equation 4}

If the two bodies are perfectly smooth, the tangential velocities of bodies will not be affected by the impulse of reaction. A rough surface, on the other hand, will affect the tangential velocities. This impulse of the reaction gives the relation between force and time for an impact with a given change of momentum as evidenced in the linear and angular impulse-momentum formulas. (Equations $2 \& 3$ )

For a given momentum change, an increased contact time will decrease the force of impact. For helmets, a stronger, more resistive, padding material in a helmet, typically expanded polystyrene (EPS), increases the distance and time of deformation under which impact kinetics occur (Figure 2). A larger deceleration distance for an equivalent velocity change reduces peak accelerations and peak forces per Newton's Second Law. Therefore, if the material for helmet padding properly matches the magnitude of impact, it will increase the $\Delta \mathrm{T}$ of the impact, decreasing the acceleration and force imparted on the skull and brain.

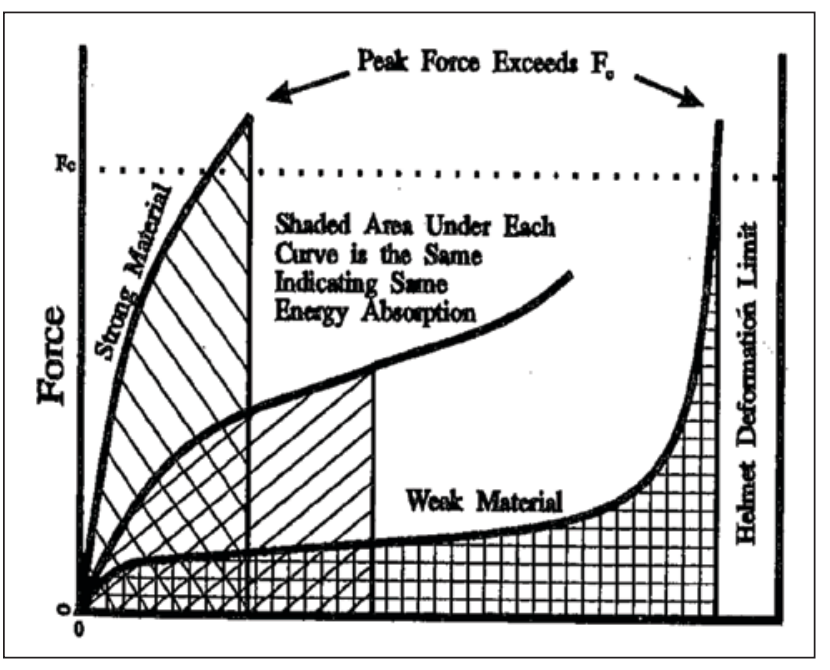

Figure 2

Force Displacement Conceptual Diagram. Effect of Padding Strength When the Same Energy is Absorbed (9)

The Head Injury Criterion, also known as HIC, is a metric commonly used by the National Highway Traffic Safety Administration and BioMedical Engineering experts to assess the potential for head injury (Equation 4). The HIC calculation empirically utilizes the integral of acceleration over time raised to the 2.5 power. For an average adult, an impact HIC value of 1000 over 36 milliseconds, or 700 over 15 milliseconds equals tolerance based on empirical data on monkeys and humans at non-injurious levels. The HIC doesn't directly account for the variations in brain mass or load direction (due to the dynamic properties of the head) or the wide variety of mechanisms of injury. However, no other head trauma or brain injury measure has been so widely generally accepted and federally adopted so for this reason, the HIC is utilized here. 


\section{Methodology}

The forensic engineering method was employed to quantify, compare and analyze the severity of impacts including energy absorption characteristics of the biological tissue with a given surface. The forensic engineering method is composed of five steps: occurrence of precedent event, define forensic engineering problem, collect data, analyze data, and develop and evaluate findings (Figure 3). Once the forensic event has occurred, for example, a nonhelmeted bicycle v. auto accident, the remaining four steps are employed and can be iteratively evaluated. Next, the instrumentation and test protocol detail for all experiments are outlined.

\section{Instrumentation and Test Protocol}

\section{for All Experiments}

A tri-axial accelerometer that collects threedimensional coordinate acceleration was mounted at the center of mass of each head-form (infant, child and adult). The acceleration data acquisition system was composed of the following components:

- Accelerometer

- Signal Conditioner

- Analog to Digital Converter

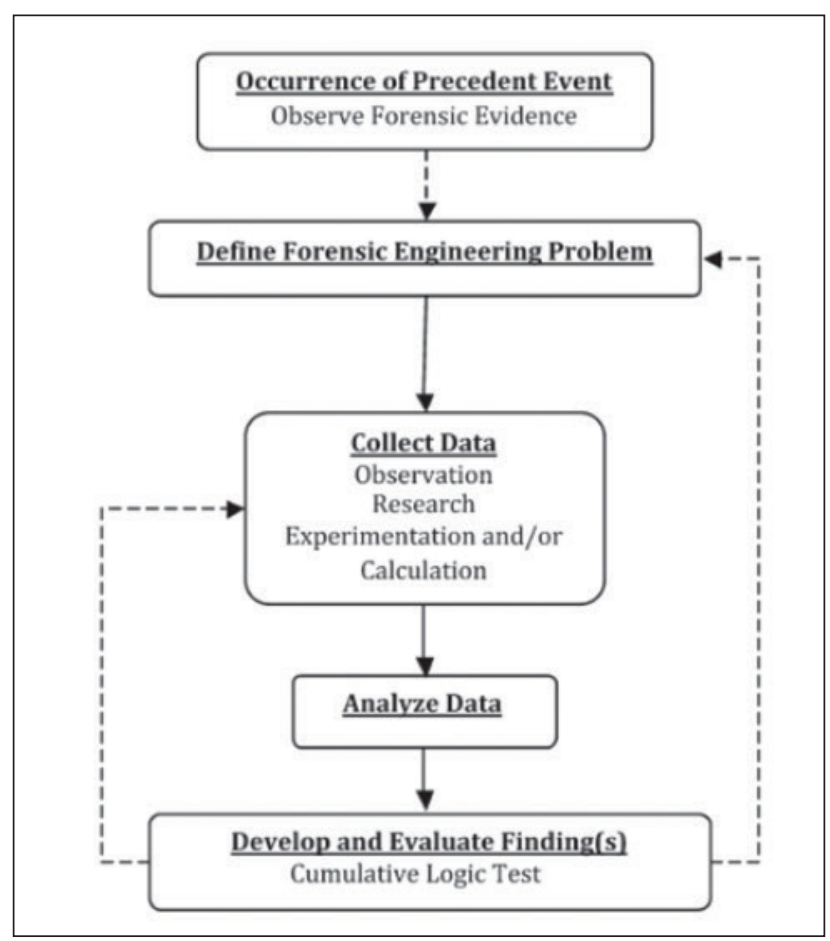

Figure 3

- DAQ Card

Acceleration data flows from the accelerometer through low noise cables to a signal conditioner; the data then flows to an analog to digital converter that transmits data to a LabView data acquisition program. Figure 4 illustrates the data acquisition flow diagram. Each channel was filtered using a 2,000 Hz anti-aliasing low pass filter and then digitally sampled at 10,000 Hz. The sensor data from the $\mathrm{x}, \mathrm{y}$, and $\mathrm{z}$ axes was filtered digitally using a 1,650 low pass filter in accordance with the SAE standard J211.

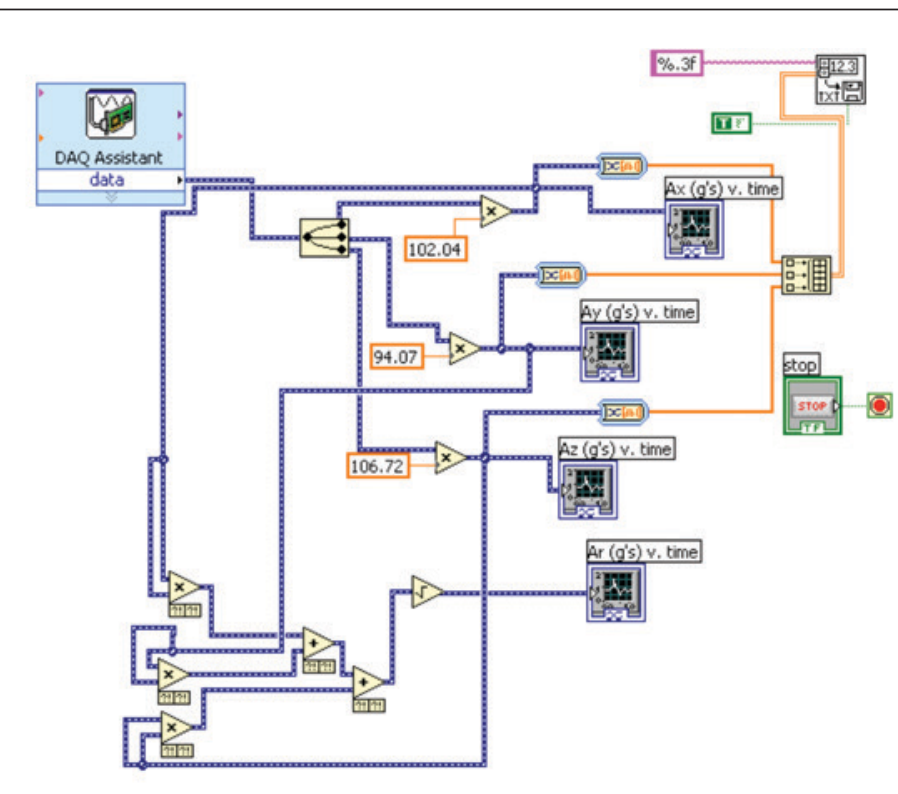

Figure 4

Digital Data Acquisition Flow Diagram

Performance is quantitatively compared by the Head Injury Criterion (HIC) as it embodies the acceleration profile and duration into a single federally standardized measure. 


\section{Head Analysis Part I:}

\section{Equestrian impacted by Sport Utility Vehicle}

A 34 year old equestrian was riding his horse across a rural highway to enter a driveway and was struck by a pickup truck at over $50 \mathrm{mph}$ (Figure 5). The horse rode up on the hood while the rider impacted the roof and fell to the ground, resulting in death from blunt force trauma including an open head wound and depressed skull fracture. The rider was not wearing any type of head protection. It is theorized that an equestrian helmet would have prevented the fatality. Our objective was to quantify the protective performance of a typical riding helmet to determine if riding helmet use would have

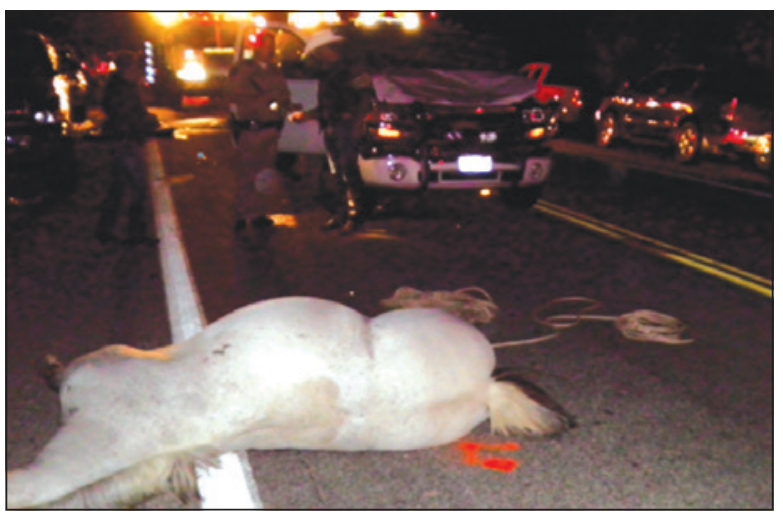

Figure 5

Scene of the Equestrian and Horse verses Sports Utility Vehicle prevented the head and brain trauma.

\section{Method}

The helmeted and non-helmeted impact tests were conducted using an inverted pendulum system consisting of a rotating rod (rods of varying length were interchanged to provide the incremental velocities). A Hybrid-III head-form and neck was mounted to the end of the rod and the helmets were fitted to the Hybrid-III head-form. When dropped, the helmeted/non-helmeted head-forms contacted an asphalt surface.

\section{Procedure}

Four exemplars were tested for each of the six helmet types at two different velocities, yielding a total of 48 impact tests. For each test, a new helmet was fitted to the head-form and the chinstrap of the helmet was secured per the manufacturer's instructions. The head-form was adjusted so that the impact would occur in the parietal/temporal/occipital region. To initiate each test, the pendulum rod with headform and helmet was raised to a $90^{\circ}$ vertical and then released. The tests were conducted at impact velocities of 20 and 25 miles per hour. Tri-axial acceleration data was collected throughout the duration of the impacts.

Results are collectively reported for all helmet types below.

\section{Helmet Analysis Part II:}

\section{Bicyclist without Helmet}

A bicyclist took a short cut through a construction zone at night, and he inadvertently fell over debris while not wearing a helmet (Figure 6). He rode his bicycle to the emergency room and reported a loss of consciousness and later claimed a brain injury. The issue here was to quantify the magnitude of the reduction in force if the bicyclist had worn a helmet when he fell. 
We tested helmets impacted onto asphalt since the Plaintiff testified that he fell and impacted the pavement, although the physical evidence on Plaintiff's face and bilateral hands was more consistent with impact into a cyclone fence that was present to close off the area (Figure 7 and 8). We included a range of test impact velocities: 5, 10, 15, 20 and $25 \mathrm{mph}$ onto asphalt.

Results compare the HIC with and without a helmet as shown in Figure 9. The helmetless head onto asphalt exceeds the HIC at lower velocities, while the helmeted head tested didn't exceed HIC until an impact speed over $20 \mathrm{mph}$. As velocity increases so does the acceleration differentiate between a helmeted and un-helmeted head.

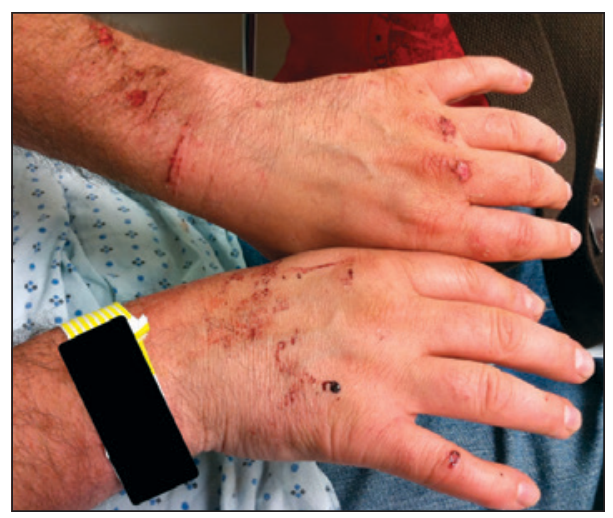

Figure 7

Trauma to Dorsal Aspect of Bilateral Hands and Wrists

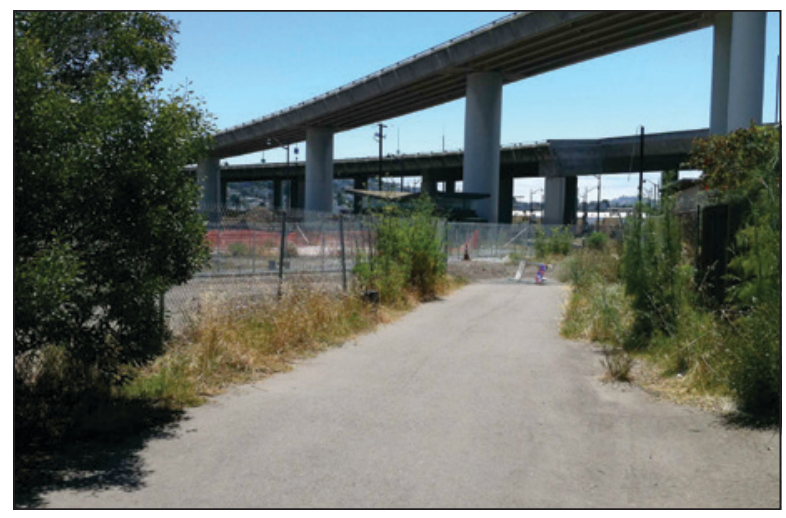

Figure 6

Scene of the Bicycle Incident

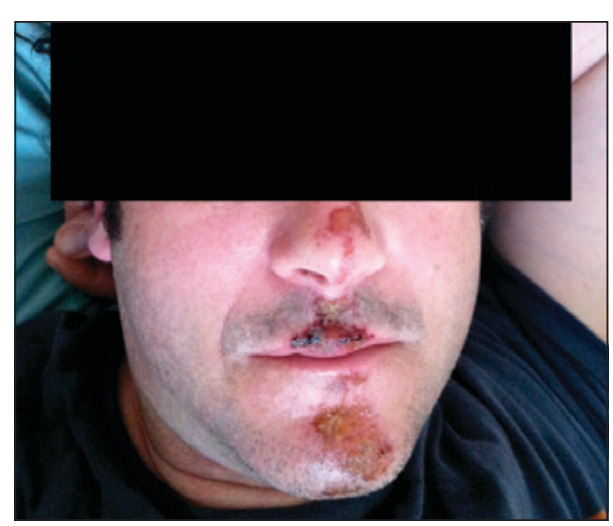

Figure 8

Trauma to the Face

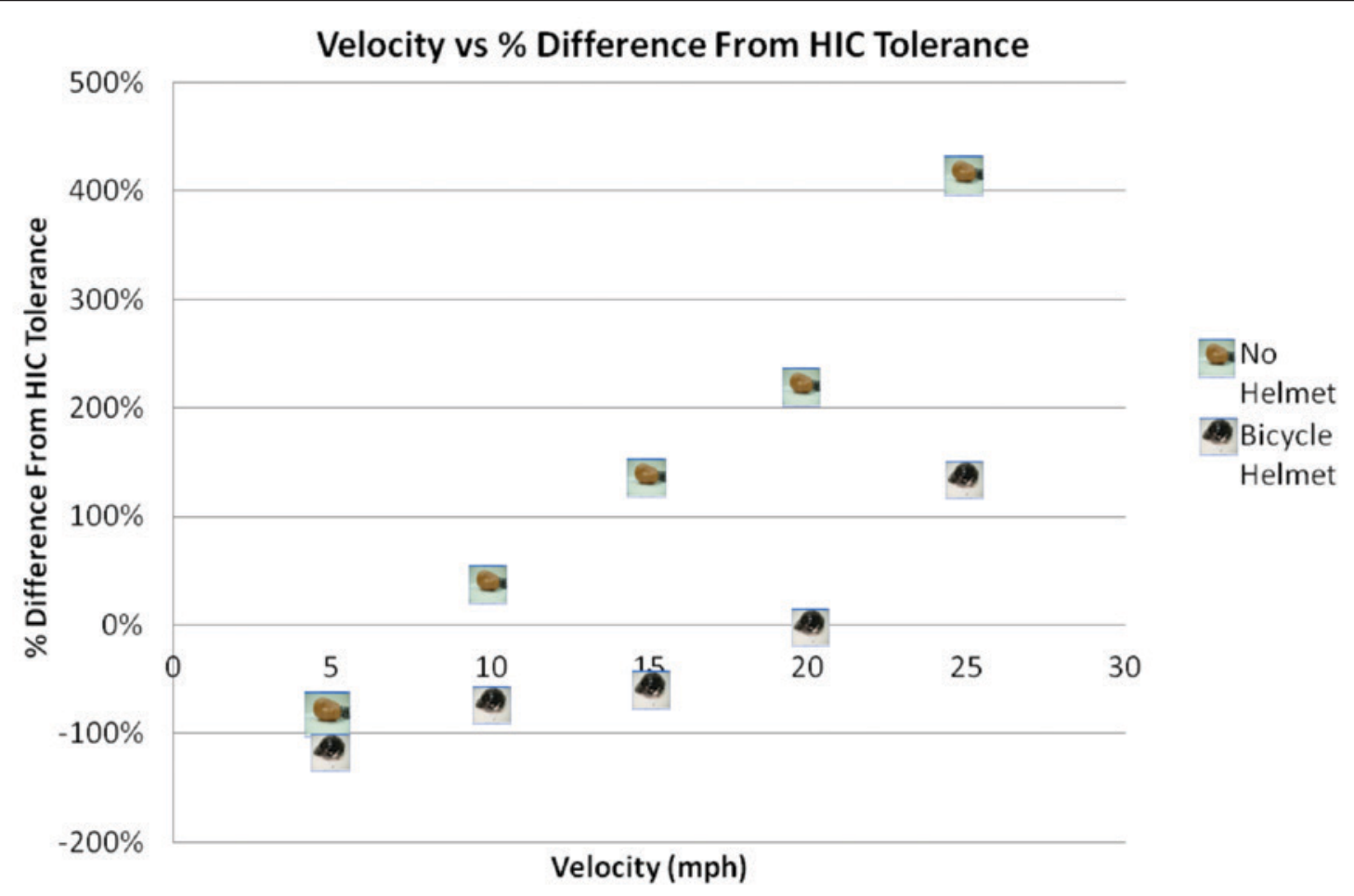

Figure 9

Bicycle Helmet Performance: Percentage Different Verses HIC Tolerance by Velocity 


\section{Helmet Analysis Part III:}

\section{Motorcyclist with Passenger into Van}

An un-helmeted motorcycle rider and passenger were waiting to proceed until it was clear on a three lane roadway. A commercial van was turning left across an intersection when witnesses reported that the motorcycle accelerated from a stop and, upon realizing there would be an impact, the operator proceeded to brake and then lay down the motorcycle (low side) in an attempt

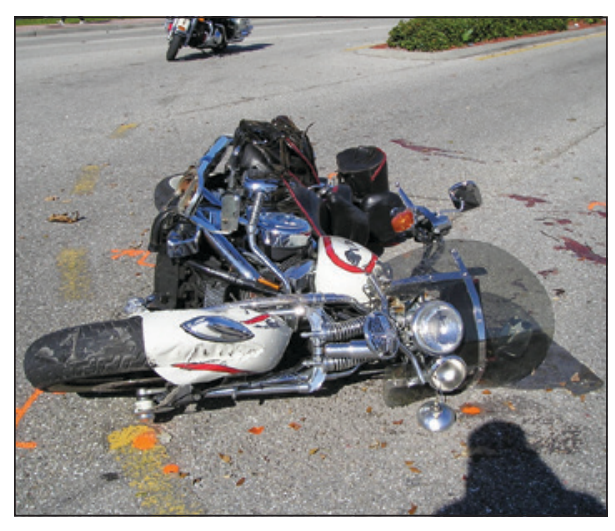

Figure 10

Motorcycle Point of Rest to avoid the collision (Figure 10). The motorcycle driver was able to skid prior to impact (Figure 11). The motorcycle impacted the side of the van, with both riders impacting the asphalt. The passenger suffered an intracranial hemorrhage with large scalp laceration and severe traumatic brain injury. Our

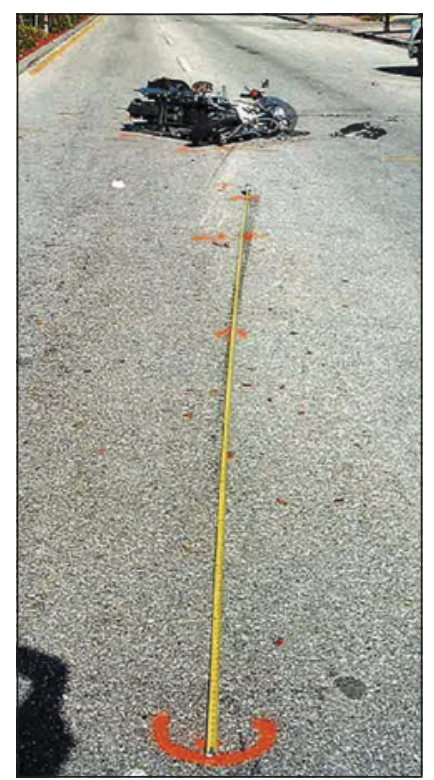

Figure 11

Motorcycle Skid and Scrape objective was to quantitatively compare the reduction in HIC if the motorcycle passenger would have been wearing a motorcycle helmet.

\section{Procedure}

Head impacts were tested at 15, 20, and $25 \mathrm{mph}$ with and without a DOT-approved full-face helmet. At all speeds tested, un-helmeted tests resulted in HIC numbers that exceeded the HIC tolerance. At 15 $\mathrm{mph}$, the helmet tested was effective in reducing HIC to below tolerance (Figure 12).

\section{Data Analysis/Results of Helmet Tests: Part I, II and III}

As seen in Figure 12, bicycle helmets outperformed many motorcycle helmets at lower velocities. This is an initially surprising result, however upon further analysis it can be understood through the selection of material and intended design. As can be seen from Figure 2, different materials are optimal for different levels of energy absorption (or speeds). If the material is too stiff the impact energy is absorbed with a lower amount of deformation and a shorter impact duration, resulting in higher levels of force. Conversely, if the material is too soft, initially the majority of impact energy is absorbed through the materials deformation over its deformable range, but towards the limit of deformation a larger portion of the remaining energy is absorbed by the head, resulting in higher levels of force. There may be a greater distance over which the impact is absorbed; however, due to the lower level of material resistance, the impact duration can be just as brief. Optimally the material will evenly absorb the impact energy over the largest distance possible, thus maximizing impact duration and minimizing peak levels of force. Each material has an ideal range of energy absorption to optimize performance. 


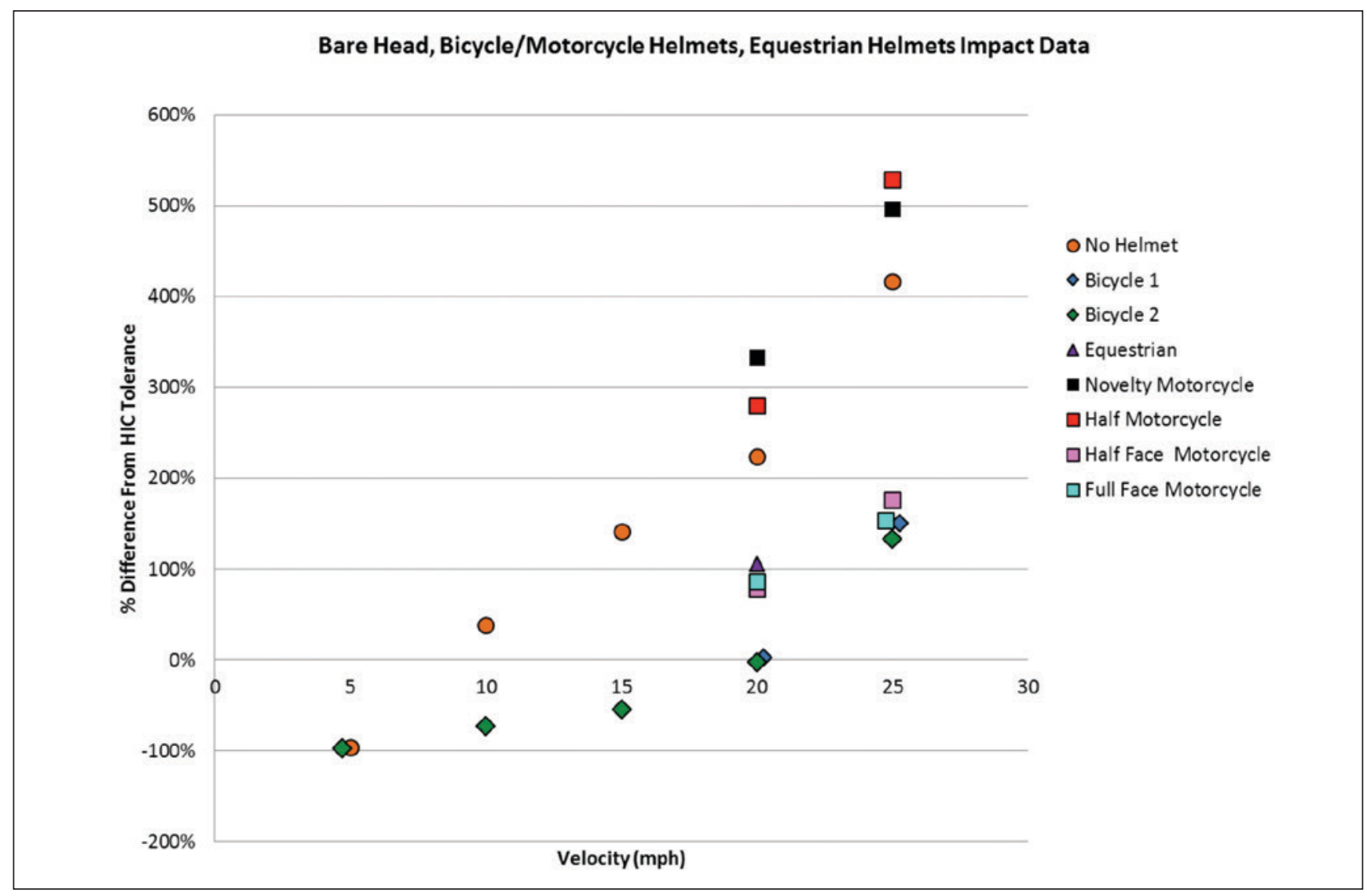

Figure 12

Motorcycle, Bicycle and Equestrian Helmet Results

It is for these reasons that performance at various speeds can vary significantly. In the case of bicycle helmets compared to motorcycle helmets, bicycle helmets are designed for lower speeds, while motorcycle helmets must cover a much larger range. Both bicycle helmets and motorcycle helmets share the design features of a thinly shelled exterior, a uniform material interior, followed by padding. The majority of the protection is provided by the uniform material of the interior, while the padding primarily aids in comfort. Thus for motorcycle helmets a single material is responsible for proving protection over a large range, and the material is likely chosen to be stiffer to provide higher levels of protection for higher energy at higher velocity. Bicycle helmets will likely have a relatively softer polystyrene material optimal for a lower range of speeds. Thus, the reason the data shows bicycle helmets outperforming motorcycle helmets is likely because the test velocities were within the optimal range of lower impact energies. From Figure 12 it can be seen that from 5-15 mph the bicycle helmet exhibits a relatively linear relationship between velocity and HIC value, while that trend breaks down from 20 to $25 \mathrm{mph}$, this could be because the impact energy levels were approaching the limit of deformation of the polystyrene liner tested. 


\section{Analysis IV:}

\section{Infant Head Impact to Tile and Carpet as well as Toy Impacts}

An unsupervised child sustained a subdural hematoma. It is theorized that since the older sister was present at the time, perhaps the older sister could have caused the trauma to the infant consistent with the older child's angry behavior. The infant was unsupervised. The older sister had been seen dropping the infant on tile and carpet as well as dropping toys into the infant's play area striking the infant. Our objective was to quantify the HIC for each of these activities to rule in or rule out the likelihood that the trauma could have been caused by these sources.

\section{Procedure}

An infant model dummy was used for all infant impact tests. In the toy series of tests, various toys were dropped onto the posterior of the infant head-form. Toys included: a keyboard, a computer, a guitar, a plastic ball, and a drum machine. In another series of tests, the model dummy was dropped from 1, 2, and $3 \mathrm{ft}$ onto a tiled surface and a carpeted surface. Tri-axial acceleration data was collected throughout the duration of every impact from the center of the infant anthropometric head form. It is theorized that the older sister could drop the child from as high as $3 \mathrm{ft}$, but the sister could drop toys from an additional foot overhead, for a total of $4 \mathrm{ft}$ drop height for the toy tests.

Table 1

Toy Weight

Toys utilized were those provided in the home. Toy weight ranged from $1.3 \mathrm{lbs}$. to $4.5 \mathrm{lbs}$. as detailed in Table 1. Results are reported in Figure 13.

\section{Data Analysis/Results of Infant Head Impact to Tile and Carpet as well as Toy Impacts}

\begin{tabular}{|c|c|}
\hline & wt (lbs) \\
\hline Ball & 1.5 \\
\hline Guita & 1.3 \\
\hline Toy Computer & 1.9 \\
\hline Drum Machine & 4.5 \\
\hline Keyboard & 2.2 \\
\hline
\end{tabular}

As shown in Figure 13, none of the activities exceeded the tolerance for head and brain injury of the infant except the 3 foot drop height onto a tile surface, which was quantified to be $76 \%$ over tolerance from 3 feet. Impacts from the toys, the lower drops onto tile as well as drops onto carpet were all so significantly under tolerance that they could be ruled out as a likely cause for the average child.

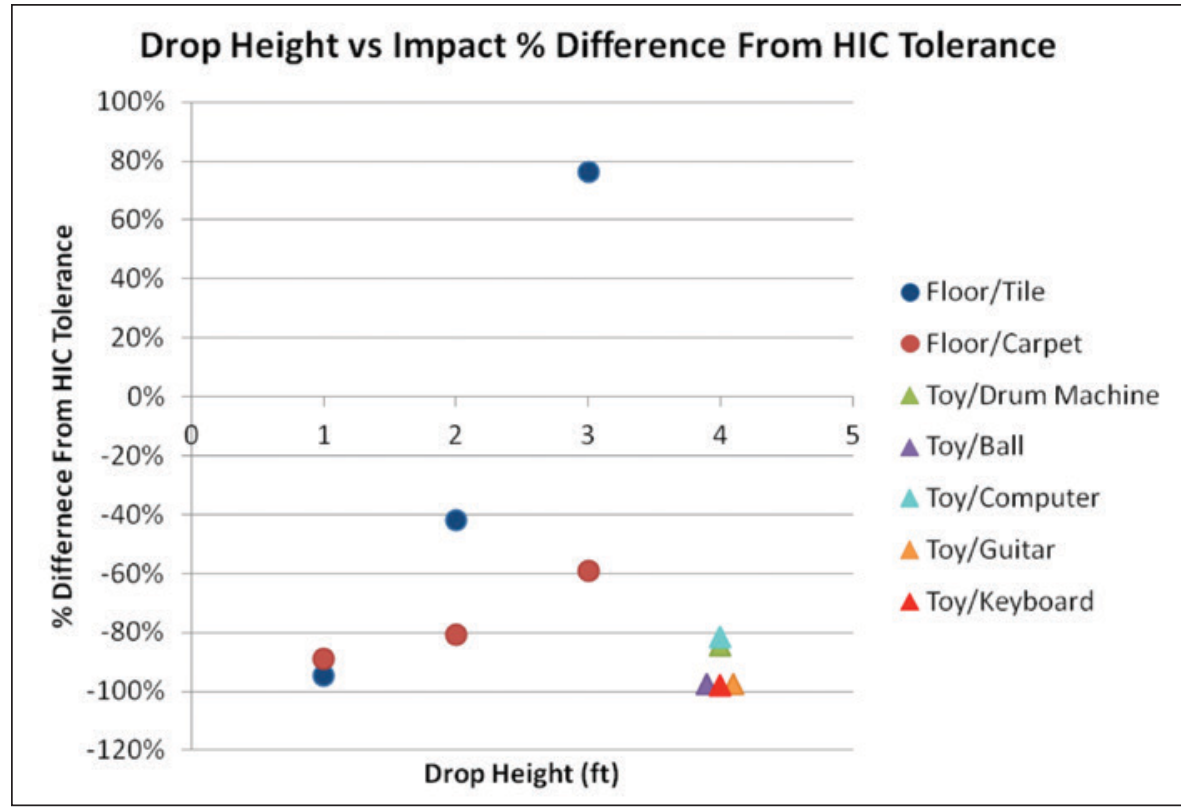

Figure 13

Infant Head Impact Test Results 


\section{Analysis V:}

\section{Ball Impact to the Child Head}

A ten year old was impacted by a playground ball. There was retinal detachment with a 10 month delayed onset of symptomatology. With a family history of retinal detachment, the objective is to assess the forces and accelerations resulting in the likelihood of retinal detachment from head impact from balls commonly used in play. Procedurally, a dummy of similar height and weight to the Plaintiff was positioned as per the Plaintiff's testimony with an impact directly to the head. Since the velocity of the ball was unknown, preliminary ball velocity kinematic tests were conducted indicating that speeds of 5-15 mph were reasonable for speed of contact. The digital data collection test was conducted by dropping four types of balls from various heights corresponding to the desired impact speeds of 5, 10, and 15 miles per hour. Three trials were conducted with each ball at each velocity. Tri-axial acceleration data was digitally collected utilizing the same protocol for all cases in this research.

\section{Data Analysis/Results of Ball Impact to the Child Head}

There appears to be a linear relationship between the velocity of the ball and the average peak level of g-forces experienced by the head-form. The dodge ball imparts the lowest amount of peak g-force during impact at the various velocities, while the baseball imparts the highest. This can be explained by the elastic modulus (stress/strain) of the balls and the distance over which the elastic modulus applies. A dodge ball is highly elastic, is capable of compressing over $20 \%$ of its width, and has a relatively low modulus of elasticity. The dodge balls' relatively low modulus of elasticity, caused by the material and the air pressure of the balls, means that a given force results in a larger amount of compression. Therefore, during an impact the ball decelerates over the span of inches and the kinetic energy is absorbed by the head over a longer duration, which reduces the amount of g-force experienced during any given period of time. On the other hand, the baseball is much more rigid, with a higher modulus of elasticity. This means that a given force leads to relatively less compression. Thus the baseball will have a shorter stopping distance and the head will absorb the baseball's kinetic energy in a shorter time duration, resulting in higher levels of acceleration. The elastic modulus characteristics of the basketball and soccer ball fall somewhere in between those of the dodge ball and the baseball, leaning towards the dodge ball due to the similar air filled interior coupled with a less elastic exterior material by comparison.

The ball impact tests show an extremely low level of g's. Since there are no tolerance values for retinal detachment, especially for those with a family history, we quantitatively compared the g's from ball impacts to normal activities of daily living. According to Bussone, while sliding down a slide a child would be expected to experience around $5.9 \mathrm{~g}$ 's. While playing jump rope around $25.7 \mathrm{~g}$ 's is typical. The dodge ball, soccer, and basketball tests impacts are comparable to the amount of g's experienced during a child's normal activities of daily living, such as sliding or playing jump rope. However, a baseball is well outside of the g's in activities of daily living. This quantitative information can then be coupled with that of a medical doctor to determine the likelihood of trauma to this specific person. 


\section{Conclusions}

The bicycle helmets outperformed all motorcycle helmets in the lower velocity tests. This is an initially surprising result, however upon further analysis it can be understood through the selection of material and intended design. There may be a greater distance over which the impact is absorbed, however due to the lower level of material resistance the impact duration can be just as brief. Optimally the material will evenly absorb the impact energy over the largest distance possible, thus maximizing impact duration and minimizing peak levels of force. Each material has an ideal range of energy absorption, and outside this range poor performance can occur.

Impacts from the toys, the lower drops onto tile as well as drops onto carpet were all so significantly under tolerance that they could be ruled out as a likely cause. It is interesting that such a mundane accident as dropping a child from 3 feet onto a non-yielding tile surface could exceed tolerance for brain injury while other accidents like a toy impact to the head proved to be trivial impacts comparatively.

For ball impacts to the head there appears to be a linear relationship between the velocity of the ball and the average peak level of g-forces experienced by the head-form. The dodge ball, soccer, and basketball tests impacts are comparable to the amount of g's experienced during a child's normal activities of daily living, such as sliding or playing jump rope. However, a baseball is well outside of the g's in activities of daily living.

These results serve to supplement head impact databases to improve mathematical modeling and the development of safety countermeasures. 


\section{References}

1. Collins, J. Accident Reconstruction. Charles C. Thomas, Springfield, IL, 1979.

2. Liptai, L., Cecil, J., Forensic Engineering and the Scientific Method. National Academy of Forensic Engineers, 2011.

3. Goldsmith, W., Impact: The Theory and Physical Behaviour of Colliding Solids. Dover Publications, Mineola, 1960.

4. Society of Automotive Engineers, Inc., J211: Instrumentation for Impact Test, SEA International, 1995.

5. Bussone, W., et al, Measurements of Non-Injurious Head Accelerations of Pediatric Population, SEA International, 2009.

6. Nahum, AM, Melvin, J. Accidental Injury: Biomechanics and Prevention. Springer, 2002.

7. MacLaughlin, T, et al. Head Impact Reconstruction - HIC Validation and Pedestrian Injury Risk. Society of Automotive Engineers 1993;930895.

8. Federal Motor Vehicle Safety Standard (FMVSS) 218, http://www.nhtsa.gov/cars/testing/comply/ fmvss218/ (accessed January 5, 2011).

9. Got, C, et al. Results of Experimental Head Impacts on Cadavers: The Various Data Obtained and Their Relations to Some Measured Physical Parameters. Society of Automotive Engineers $1978 ; 780887$.

10. Liptai, L., Forensic Engineering Analysis of DOT verses Non-DOT Helmet Performance as a Function of Velocities Exceeding Standardized Testing, National Academy of Forensic Engineers, 2013. 